\title{
Elevated $\mathrm{HbA}_{1 \mathrm{c}}$ levels and the accumulation of differentiated $\mathrm{T}$ cells in $\mathrm{CMV}^{+}$individuals
}

\author{
Jerrald L. Rector ${ }^{1,2}$ • G. Neil Thomas ${ }^{3}$ - Victoria E. Burns ${ }^{1}$ • Jennifer B. Dowd ${ }^{4,5}$. \\ Raphael M. Herr ${ }^{2}$ • Paul A. Moss ${ }^{6} \cdot$ Marc N. Jarczok $^{2} \cdot$ Kristina Hoffman $^{2}$ • \\ Joachim E. Fischer ${ }^{2}$. Jos A. Bosch ${ }^{2,7}$
}

Received: 30 April 2015 / Accepted: 23 July 2015 / Published online: 20 August 2015

(C) The Author(s) 2015. This article is published with open access at Springerlink.com

\begin{abstract}
Aims/hypothesis Biological ageing of the immune system, or immunosenescence, predicts poor health and increased mortality. A hallmark of immunosenescence is the accumulation of differentiated cytotoxic $\mathrm{T}$ cells $\left(\mathrm{CD} 27^{-} \mathrm{CD} 45 \mathrm{RA}^{+-}\right.$; or dCTLs), partially driven by infection with the cytomegalovirus (CMV). Immune impairments reminiscent of immunosenescence are also observed in hyperglycaemia, and in vitro studies have illustrated mechanisms by which elevated glucose can lead to increased dCTLs. This study explored associations between glucose dysregulation and markers of immunosenescence in $\mathrm{CMV}^{+}$and $\mathrm{CMV}^{-}$ individuals.
\end{abstract}

Electronic supplementary material The online version of this article (doi:10.1007/s00125-015-3731-4) contains peer-reviewed but unedited supplementary material, which is available to authorised users.

Jos A. Bosch

j.a.bosch@uva.nl

1 School of Sport, Exercise, and Rehabilitation Sciences, University of Birmingham, Birmingham, UK

2 Mannheim Institute of Public Health, Social and Preventive Medicine, Mannheim Medical Faculty, University of Heidelberg, Mannheim, Germany

3 School of Health and Population Sciences, College of Medical and Dental Sciences, University of Birmingham, Birmingham, UK

4 CUNY School of Public Health, New York, NY, USA

5 CUNY Institute for Demographic Research, New York, NY, USA

6 Cancer Research UK Centre, University of Birmingham, Birmingham, UK

7 Department of Psychology, University of Amsterdam, Weesperplein 4, 1018 XA Amsterdam, The Netherlands
Methods A cross-sectional sample of participants from an occupational cohort study $(n=1,103$, mean age 40 years, $88 \%$ male) were assessed for $\mathrm{HbA}_{1 \mathrm{c}}$ and fasting glucose levels, diabetes, cardiovascular risk factors (e.g. lipids), numbers of circulating effector memory (EM; $\mathrm{CD} 27^{-} \mathrm{CD} 45 \mathrm{RA}^{-}$) and CD45RA re-expressing effector memory (EMRA; $\mathrm{CD} 27^{-} \mathrm{CD}^{2} 5 \mathrm{RA}^{+}$) T cells, and CMV infection status. Selfreport and physical examination assessed anthropometric, sociodemographic and lifestyle factors.

Results Among $\mathrm{CMV}^{+}$individuals $(n=400)$, elevated $\mathrm{HbA}_{1 \mathrm{c}}$ was associated with increased numbers of EM $(B=2.75$, $p<0.01)$ and EMRA $(B=2.90, p<0.01) \mathrm{T}$ cells, which was robust to adjustment for age, sex, sociodemographic variables and lifestyle factors. Elevated EM T cells were also positively associated with total cholesterol $(B=0.04, p<0.05)$ after applying similar adjustments. No associations were observed in $\mathrm{CMV}^{-}$individuals.

Conclusions/interpretation The present study identified consistent associations of unfavourable glucose and lipid profiles with accumulation of dCTLs in $\mathrm{CMV}^{+}$individuals. These results provide evidence that the impact of metabolic risk factors on immunity and health can be co-determined by infectious factors, and provide a novel pathway linking metabolic risk factors with accelerated immunosenescence.

Keywords Cholesterol · CMV · Cytomegalovirus · Diabetes · Glucose $\cdot$ Haemoglobin $\mathrm{A}_{1 \mathrm{c}} \cdot \mathrm{HbA}_{1 \mathrm{c}} \cdot$ Immune ageing $\cdot$ Metabolic syndrome $\cdot$ T cell

$\begin{array}{ll}\text { Abbreviations } \\ \text { APC } & \text { Allophycocyanin } \\ \text { CMV } & \text { Cytomegalovirus } \\ \text { CVD } & \text { Cardiovascular disease } \\ \text { Cy7 } & \text { Cyanine dye } 7\end{array}$

Abbreviations

CMV Cytomegalovirus

Cy7 Cyanine dye 7 


$\begin{array}{ll}\text { dCTL } & \text { Differentiated cytotoxic T cell } \\ \text { EM } & \text { Effector memory } \\ \text { EMRA } & \text { CD45RA re-expressing effector memory } \\ \text { HDL-C } & \text { HDL-cholesterol } \\ \text { LDL-C } & \text { LDL-cholesterol } \\ \text { PE } & \text { Phycoerythrin } \\ \text { SES } & \text { Socioeconomic status }\end{array}$

\section{Introduction}

The progressive impairment of immunity with age, known as immunosenescence, is thought to underlie increased infection risk and mortality $[1,2]$ and may also contribute to several other age-associated complications, including low-grade inflammation and increased cardiovascular disease (CVD) risk [3-5]. Infection with cytomegalovirus (CMV) has been shown to accelerate features of immunosenescence $[2,6-8]$. This herpes virus establishes a lifelong latent infection, interrupted by periods of non-clinical reactivation. The resultant activation of CMV-specific $\mathrm{T}$ cells leads to a marked accumulation of differentiated cytotoxic T cells (dCTLs; $\mathrm{CD} 27^{-} \mathrm{CD}^{2} 5 \mathrm{RA}^{+/}$), which can be subdivided into effector memory (EM; CD27 ${ }^{-} \mathrm{CD}^{2} \mathrm{RAA}^{-}$) $\mathrm{T}$ cells and CD45RA reexpressing EM T cells (EMRA; CD27- ${ }^{-}$D45RA ${ }^{+}$) $[6,9]$. Indeed, infected (i.e. $\mathrm{CMV}^{+}$) individuals have on average three- to fourfold higher dCTL numbers compared with uninfected (i.e. CMV) individuals, although large inter-individual differences exist [10].

The accumulation of dCTLs may make a material contribution to the acceleration of immunosenescence [11] and is thought to provide a mechanism through which immunosenescence may be associated with health outcomes, such as CVD [12]. For example, these T cells show a high production of proinflammatory cytokines, have short telomeres and have an aberrant proliferative capacity [13, 14]. Thus, the accumulation of dCTLs may be the mechanism linking CMV with the hallmarks of immune ageing.

Significantly, many of the immune system impairments that have been associated with ageing resemble those of chronic hyperglycaemia. For example, impaired glucose tolerance and diabetes are associated with poor control of infection [15-17], impaired vaccination responses [18], elevated inflammatory activity [19] and shorter leucocyte telomere length $[4,20]$. These observations raise the question of whether the immune effects of hyperglycaemia may, at least in part, involve the accumulation of dCTLs [21]. For example, in vitro studies show that strong $\mathrm{T}$ cell stimulation - similar to that which might be elicited by CMV reactivation - enhances cellular glucose uptake, which can lead to the accumulation of readily activated memory $T$ cells that acquire resistance to cell death [22]. This presents a potential mechanism whereby hyperglycaemia may amplify the CMV-induced accumulation of dCTLs.

Therefore, the aim of the current study was to examine the relationship between glucose metabolism (i.e. $\mathrm{HbA}_{1 \mathrm{c}}$ and fasting glucose levels and diabetic status) and EM and EMRA T cell numbers in a large sample of $\mathrm{CMV}^{+}$and $\mathrm{CMV}^{-}$individuals. It was hypothesised that the effects of CMV infection on dCTL numbers would be enhanced in $\mathrm{CMV}^{+}$individuals that show evidence of elevated glucose. Additionally, other factors associated with hyperglycaemia that may contribute to increased $\mathrm{dCTL}$ accumulation were also examined, including markers of dyslipidaemia (i.e. elevated circulating triacylglycerol and LDL-cholesterol [LDL-C], and lower HDL-cholesterol [HDL-C]) and elements of the metabolic syndrome [23].

\section{Methods}

Participants The present study was conducted among employees $(n=1,103 ; 88 \%$ male; mean age 40 years [range 18 64 years]) of a large European airplane manufacturer in the south of Germany who took part in a voluntary company health check in 2011. Participant characteristics are presented in Table 1. Participants received a personalised comprehensive health report. All data were anonymised before analysis. This study was approved by the ethics committee of the Medical Faculty Mannheim, Heidelberg University. All participants gave written informed consent.

Procedures Participants arrived at a location away from their usual workplace between 06:45 and 08:45 hours in the morning for their health check. After drawing fasting venous blood and a medical examination, participants were seated in a quiet room to fill out questionnaires on demographic, medical and health behaviour data. Anthropometric (e.g. height, weight, waist and hip circumference) and BP measurements were carried out by trained study personnel. Demographic data, including age, sex and marital status, along with socioeconomic status (SES) indicators (measured as hierarchical job position, manual occupation and shift work), self- and doctordiagnosed medical conditions, and lifestyle factors (e.g. smoking, alcohol intake, exercise) were obtained by questionnaires used and validated in the MONICA ('Monitoring trends and determinants in cardiovascular disease') study [24].

Flow cytometry $\mathrm{T}$ cell phenotypes were assessed by flow cytometry. Whole blood samples were collected in EDTAcoated tubes (Sarstedt, Nümbrecht, Germany), stored at room temperature and prepared within $1 \mathrm{~h}$ of collection. Briefly, $30 \mu \mathrm{l}$ whole blood was stained with a combination of the following conjugated monoclonal antibodies: anti-CD3 allophycocyanin (APC)-cyanine dye 7 (Cy7; clone SK7), 
Table 1 Participant sociodemographic and lifestyle characteristics

\begin{tabular}{|c|c|c|c|c|}
\hline \multirow[t]{2}{*}{ Characteristic } & \multirow[t]{2}{*}{ Total } & \multicolumn{2}{|l|}{ CMV status } & \multirow[t]{2}{*}{$p$ value } \\
\hline & & Positive & Negative & \\
\hline$n(\%)$ & 1,103 & $400(36)$ & $703(64)$ & \\
\hline Age (years) & $40.1 \pm 11.0$ & $41.5 \pm 11.1$ & $39.3 \pm 10.8$ & $<0.001$ \\
\hline Sex $(\%$ male $)$ & 87.7 & 84.5 & 89.5 & 0.020 \\
\hline Married/co-habiting (\%) & 77.0 & 80.4 & 75.0 & 0.065 \\
\hline Job status $(\%)$ & - & - & - & 0.007 \\
\hline Division/dept mgr & 4.8 & 3.5 & 5.6 & - \\
\hline Project leader/process mgr & 15.3 & 13.9 & 16.2 & - \\
\hline Worker (mgrl) & 6.7 & 6.0 & 7.1 & - \\
\hline Skilled worker (non-mgrl) & 63.8 & 62.6 & 64.4 & - \\
\hline Semi-skilled worker & 9.4 & 14.1 & 6.7 & - \\
\hline Shift worker (\% yes) & 28.1 & 33.8 & 24.9 & 0.003 \\
\hline Manual occupation (\% yes) & 49.6 & 55.0 & 46.5 & 0.008 \\
\hline Smoking (\%) & - & - & - & 0.050 \\
\hline Never smoker & 46.1 & 41.1 & 48.9 & - \\
\hline Former smoker & 24.9 & 27.0 & 23.8 & - \\
\hline Smoker & 29.0 & 31.9 & 27.3 & - \\
\hline Cigarettes per day (in smokers) & $14 \pm 8$ & $15 \pm 7$ & $13 \pm 8$ & 0.090 \\
\hline Alcohol (\%) & - & - & - & $<0.001$ \\
\hline $0-2$ times/month & 24.9 & 30.4 & 21.7 & - \\
\hline 1-2 times/week & 29.9 & 32.5 & 28.5 & - \\
\hline 3-7 times/week & 45.2 & 37.2 & 49.8 & - \\
\hline Leisure physical activity (h/week) & $7.0 \pm 7.5$ & $7.1 \pm 9.4$ & $7.0 \pm 6.2$ & 0.307 \\
\hline
\end{tabular}

Data are unadjusted comparisons of participant characteristics: a Student's $t$ test was performed for continuous variables and a $\chi^{2}$ test for categorical variables

Data are means \pm SD unless otherwise stated

Dept, department; mgr, manager; mgrl, managerial

anti-CD4-peridinin chlorophyll protein (clone SK3), anti- $\gamma \delta$ $\mathrm{T}$ cell receptor $(\gamma \delta \mathrm{TCR})$-phycoerythrin (PE; clone B1), antiCD8-APC (clone SK1; BD Biosciences, San José, CA, USA); and anti-CD45RA-FITC (clone HI100) and antiCD27-PE-Cy7 (clone M-T271; BD Pharmingen, San Diego, CA). All antibodies were purchased from and validated by BD Biosciences and BD Pharmingen at pre-diluted concentrations for use at the recommended volume per test. Following a $20 \mathrm{~min}$ incubation at room temperature in the dark, $1.5 \mathrm{ml}$ BD FACS lysing solution (BD Biosciences) was added to the mixture and incubated for another $15 \mathrm{~min}$. After centrifugation for $7 \mathrm{~min}$ at $700 \mathrm{~g}$, the supernatant was removed and both lysed erythrocytes and unbound antibody were washed away. The pellet was subsequently re-suspended in $250 \mu 12 \%$ paraformaldehyde solution until analysis. Data were collected using a FACSCanto II flow cytometer and dedicated FACSDiva software (BD Biosciences). Spectral overlap was electronically compensated for using single labelled antibody tubes. Following data acquisition, files were transferred to a third party software program (FlowJo v7.6.5, Tree Star,
Ashland, OR, USA) for analysis. Representative plots of the gating strategy are shown in electronic supplementary material Fig. 1. Lymphocyte numbers were obtained by multiplying the total leucocyte count by the percentage of gated lymphocytes. The lymphocyte number was further multiplied by the percentages of gated $\mathrm{CD}^{+}$cells and their subsequent subsets to calculate the numbers of cells per microlitre used in the analyses.

CMV status determination Fasting plasma samples were stored in small aliquots at $-80^{\circ} \mathrm{C}$ until analysis. Evidence of a previous CMV infection (serostatus) was determined using a commercially available ELISA (BioCheck, Foster City, CA, USA) according to the manufacturer's instructions. Optical density values obtained from participants' samples were fitted to a standard curve. These concentrations were then compared with a cut-off value to compute CMV index scores. Participants with a borderline seropositive result, i.e. a calculated index score of $>0.85$ and $<1.15$, were re-tested $(n=9)$. If they remained borderline, participants with index scores 
above and below 1.00 were considered $\mathrm{CMV}^{+}$and $\mathrm{CMV}^{-}$, respectively, as per the manufacturer's instructions. The sensitivity, specificity and accuracy of the test are reported as $95.0 \%, 96.7 \%$ and $96.0 \%$, respectively.

Biochemical analysis $\mathrm{HbA}_{1 c}$, fasting glucose, triacylglycerol, LDL-C, HDL-C and high-sensitivity C-reactive protein levels were measured by an accredited clinical laboratory (Synlab Laboratories, Augsburg, Germany) according to standard laboratory procedures that comply with International Organization for Standardization norms (DIN EN ISO 15189). $\mathrm{HbA}_{1 \mathrm{c}}$ levels were measured using a secondgeneration $\mathrm{HbA}_{1 \mathrm{c}}$ immunoassay (Roche Diagnostics, Mannheim, Germany), and fasting glucose levels were measured using the glucose hexokinase enzymatic assay (Glucose OSR6121, Beckman Coulter, Brea, CA, USA), in accordance with the latest standardised guidelines and recommendations for laboratory analysis in the diagnosis of diabetes [25]. Cholesterol and triacylglycerol were automatically measured enzymatically (Cobas 8000 analyser; Roche Diagnostics). HDL-C was measured using a competitive homogeneous assay (Roche Diagnostics), and LDL-C was calculated using the Friedewald equation [26]. These values were also used to calculate the ratio of LDL-C to HDL-C.

Diabetes and metabolic syndrome classification Diabetes was classified according to the ADA guidelines in individuals with fasting glucose levels of $>6.94 \mathrm{mmol} / \mathrm{l}$ and/or $\mathrm{HbA}_{1 \mathrm{c}}$ levels of $\geq 6.5 \%$ ( $48 \mathrm{mmol} / \mathrm{mol})$ in the absence of known diabetes. Those with self-reported, doctor-diagnosed diabetes were also classified as diabetic. Prediabetes was classified as a fasting glucose level between 5.55 and $6.94 \mathrm{mmol} / \mathrm{l}$ and/or an $\mathrm{HbA}_{1 \mathrm{c}}$ level between $5.7 \%$ (39 $\left.\mathrm{mmol} / \mathrm{mol}\right)$ and $6.4 \%$ (46 mmol/mol) [27]. The remaining normal-glycaemic individuals, therefore, had fasting glucose and $\mathrm{HbA}_{1 \mathrm{c}}$ levels of $<5.55 \mathrm{mmol} / 1$ and $<5.7 \%$, respectively. The metabolic syndrome components were assessed as the following: (1) waist circumference $>102 \mathrm{~cm}$ (men) or $>88 \mathrm{~cm}$ (women); (2) plasma triacylglycerol $>1.70 \mathrm{mmol} / \mathrm{l}$; (3) plasma HDL-C $<1.03 \mathrm{mmol} / 1$ (men) or $<1.29 \mathrm{mmol} / 1$ (women); (4) BP $\geq 130 \mathrm{mmHg}$ (systolic) and/or $\geq 85$ (diastolic) $\mathrm{mmHg}$; and (5) plasma fasted glucose $\geq 5.55 \mathrm{mmol} / \mathrm{l}$. Each of these components were dichotomised (yes or no) and added together to create a metabolic syndrome component score (range $0-5$ ). Those with a score of $\geq 3$ were classified as having the metabolic syndrome [28].

Statistical analysis To approximate a normal distribution of the variables used in the current analyses, we applied transformations based on information criteria obtained from the Ladder-of-Powers in Stata 12 (StataCorp, College Station, TX, USA). The transformation with the least statistical deviation from a normal distribution, indicated by the smallest $\chi^{2}$ (or the most non-significant $p$ value) was used, as previously recommended [29]. Missing data ( $<7 \%$ for all variables) was handled by multiple imputation in IBM SPSS (version 20, Chicago, IL, USA). Briefly, a fully conditional specification method was automatically chosen to replace missing data. In this method, each variable was fitted in a univariate (single dependent variable) model using all other available variables in the model as predictors, and missing values were imputed for each variable being fitted. Linear and logistic regressions were used for continuous and categorical variables, respectively. Relevant variables with already complete data were entered only as predictors to improve estimates. After ten iterations for each of the five imputation datasets, pooled estimates were used for all subsequent analyses below.

First, participant characteristics (i.e. demographics and lifestyle behaviours) were compared between $\mathrm{CMV}^{+}$and $\mathrm{CMV}^{-}$ individuals. Student's $t$ tests and $\chi^{2}$ analyses were used for continuous and categorical variables, respectively.

Second, differences in CMV status by $\mathrm{HbA}_{1 \mathrm{c}}$ and fasting glucose levels and diabetic status were explored using binary logistic regressions. CMV status was entered as the dependent variable, and each factor was entered, in turn, as an independent variable. Potential confounders known to impact CMV infection and reactivation, including age, sex, marital status, SES (job status, manual occupation), and lifestyle factors (smoking, alcohol intake, BMI, and physical activity) [30-32], were statistically controlled in hierarchical models (Models 1-3): Model 1 was adjusted for age and sex; Model 2 was Model 1 additionally adjusted for marital status and SES (job status and manual occupation); and Model 3 was Model 2 further adjusted for smoking, alcohol, BMI and physical activity. These models were entered stepwise as covariates throughout the remaining analyses.

Third, numbers of $\mathrm{CD} 8^{+} \mathrm{EM}$ and EMRA T cells were compared between levels of glycaemic control (indicated by diabetic classification) using ANOVA and ANCOVA. These analyses were stratified by CMV status and the abovementioned potential confounders were entered as covariates (Models 1-3).

Finally, separate linear regressions were used to explore the individual associations of $\mathrm{HbA}_{1 \mathrm{c}}$ and fasting glucose levels with EM and EMRA T cell subset numbers. Potential confounders were entered as covariates using the same models as above.

The above analyses were repeated with each of the dyslipidaemia and CVD risk factors (i.e. total cholesterol, LDL-C, HDL-C, the LDL-C to HDL-C ratio and triacylglycerol) entered separately as independent predictors of EM and EMRA T cell subset numbers. For significant associations, $\mathrm{HbA}_{1 \mathrm{c}}$ was added as a potential mediator to examine the role of glucose levels on lipid metabolism. All analyses were performed with IBM SPSS version 20. 


\section{Results}

Participant characteristics As shown in Table 1, 400 of the $1,103(36.3 \%)$ participants were $\mathrm{CMV}^{+}$. On average, $\mathrm{CMV}^{+}$ participants tended to be older and female. They were also more likely to be current or former smokers, to drink less frequently, and to have a lower SES (low job status, more manual occupations and shift work). There was no difference in the amount of cigarettes smoked (among smokers), BMI, WHR or physical activity ( $p>0.10$ for all; Table 1$)$. The tabulation of metabolic risk factors revealed that 290 (26.3\%) individuals met the criteria for metabolic syndrome classification. Regarding diabetes, 663 were classified as normal, 404 as prediabetic and 36 as diabetic. Because of the small number of diabetic individuals, the diabetic group was merged with the prediabetic group and labelled 'hyperglycaemic'.

Glycaemic control and dyslipidaemia factors are associated with CMV infection Unadjusted analyses showed that $\mathrm{CMV}^{+}$individuals were more likely to have higher levels of $\mathrm{HbA}_{1 \mathrm{c}}(38.1$ vs $37.7 \mathrm{mmol} / \mathrm{mol})$ and to be classified as hyperglycaemic, i.e. prediabetic or diabetic $(46.2 \%$ vs
36.4\%; Table 2). In binary logistic regressions, the associations of $\mathrm{HbA}_{1 \mathrm{c}}$ and hyperglycaemic status with CMV infection status were reduced to non-significance after adjusting for age and sex (Model 1) and sociodemographic factors (Model 2), respectively.

Unadjusted comparisons of dyslipidaemia factors between $\mathrm{CMV}^{+}$and $\mathrm{CMV}^{-}$individuals revealed that $\mathrm{CMV}^{+}$individuals were also more likely to have HDL-C levels that fell within the metabolic syndrome classification range (men $<1.03 \mathrm{mmol} / \mathrm{l}$, women $<1.29 \mathrm{mmol} / \mathrm{l})$. However, none of the other metabolic characteristics, including the metabolic syndrome classification, differed by CMV status in unadjusted analyses (Table 2). After progressive adjustment for possible confounders (Models 1-3), the association with the low HDL-C category was no longer significant, but increased levels of continuous HDL-C became significantly associated with CMV infection ((OR 0.55 $[95 \% \mathrm{CI}] 0.319-0.960), p=0.035)$. That is, individuals with higher HDL-C levels (natural log-transformed) had a lower risk of CMV infection.

Glycaemic control and its interaction with CMV infection are associated with dCTL numbers Fig. 1 shows the
Table 2 Participant metabolic characteristics

\begin{tabular}{|c|c|c|c|c|}
\hline \multirow[t]{2}{*}{ Characteristic } & \multirow[t]{2}{*}{ Total } & \multicolumn{2}{|l|}{ CMV status } & \multirow[t]{2}{*}{$p$ value } \\
\hline & & Positive & Negative & \\
\hline$n(\%)$ & $1,103(100)$ & $400(36)$ & $703(64)$ & \\
\hline $\mathrm{HbA}_{1 \mathrm{c}}(\%)$ & $5.61 \pm 0.01$ & $5.64 \pm 0.02$ & $5.60 \pm 0.01$ & 0.047 \\
\hline $\mathrm{HbA}_{1 \mathrm{c}}(\mathrm{mmol} / \mathrm{mol})$ & $37.84 \pm 0.11$ & $38.13 \pm 0.18$ & $37.67 \pm 0.13$ & 0.060 \\
\hline Fasting glucose $(\mathrm{mmol} / \mathrm{l})$ & $4.82 \pm 0.02$ & $4.85 \pm 0.03$ & $4.80 \pm 0.02$ & 0.168 \\
\hline Total cholesterol (mmol/l) & $5.25 \pm 0.03$ & $5.28 \pm 0.05$ & $5.24 \pm 0.04$ & 0.591 \\
\hline LDL-C (mmol/l) & $3.20 \pm 0.03$ & $3.22 \pm 0.04$ & $3.19 \pm 0.03$ & 0.580 \\
\hline HDL-C (mmol/l) & $1.40 \pm 0.01$ & $1.38 \pm 0.02$ & $1.41 \pm 0.02$ & 0.068 \\
\hline LDL-C/HDL-C ratio & $2.47 \pm 0.03$ & $2.54 \pm 0.05$ & $2.42 \pm 0.04$ & 0.140 \\
\hline Triacylglycerol (mmol/l) & $1.42 \pm 0.03$ & $1.47 \pm 0.05$ & $1.39 \pm 0.03$ & 0.145 \\
\hline Diabetes classification (\%) & - & - & - & 0.005 \\
\hline Normal & 60.1 & 53.9 & 63.6 & - \\
\hline Prediabetes & 36.6 & 41.9 & 33.7 & - \\
\hline Diabetes & 3.3 & 4.3 & 2.7 & - \\
\hline Metabolic syndrome ( $\%$ yes) & 26.3 & 28.0 & 25.3 & 0.368 \\
\hline Waist circumference ( $\%$ yes) & 52.1 & 54.0 & 51.0 & 0.387 \\
\hline Triacylglycerol (\% yes) & 31.8 & 35.0 & 30.0 & 0.101 \\
\hline HDL-C (\% yes $)$ & 17.7 & 21.0 & 15.8 & 0.036 \\
\hline BP $(\%$ yes $)$ & 52.5 & 49.5 & 54.2 & 0.150 \\
\hline Fasting glucose (\% yes) & 10.0 & 11.0 & 9.4 & 0.442 \\
\hline BMI, $\left(\mathrm{kg} / \mathrm{m}^{2}\right)$ & $24.46 \pm 4.10$ & $24.46 \pm 4.13$ & $24.46 \pm 3.98$ & 0.929 \\
\hline WHR & $0.90 \pm 0.08$ & $0.90 \pm 0.08$ & $0.90 \pm 0.07$ & 0.190 \\
\hline
\end{tabular}

All are unadjusted comparisons of participant metabolic characteristics: a Student's $t$ test was performed for continuous variables and a $\chi^{2}$ test for categorical variables. HDL-C is natural log-transformed, LDL-C is square root-transformed and total cholesterol is square root-transformed

Values are means \pm SD unless otherwise stated 


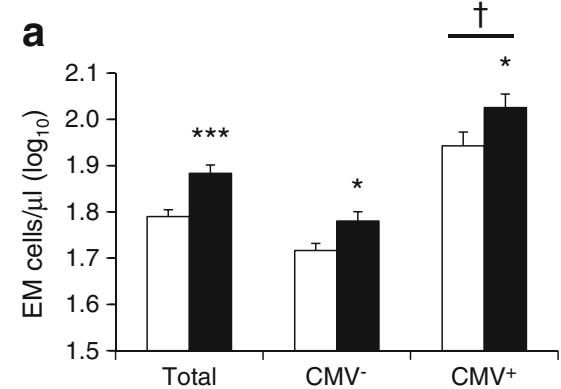

Fig. 1 Unadjusted comparison of (a) EM (CD27 $\left.{ }^{-} \mathrm{CD}^{2} 5 \mathrm{RA}^{-}\right)$and (b) EMRA (CD27 ${ }^{-}$D45RA ${ }^{+}$) $\mathrm{CD}^{+} \mathrm{T}$ cell subset numbers by glycaemic status and CMV infection. White bars, normoglycaemic; black bars,

unadjusted comparisons of EM and EMRA T cell numbers stratified by glycaemic status and CMV infection. For all participants, individuals classified as hyperglycaemic had $26.6 \%$ higher numbers of EM (110.2 vs $87.0 \mathrm{cells} / \mu \mathrm{l})$ and $41.2 \%$ higher EMRA T cells $(218.1$ vs 154.5 cells $/ \mu 1$; both $p<0.001$ ) than normoglycaemic participants.

When further stratified by CMV status, the results showed significantly more EMRA T cells in hyperglycaemic vs normoglycaemic $\mathrm{CMV}^{+}$individuals $(p<0.001)$, while no such difference was observed in the $\mathrm{CMV}^{-}$group (Fig. 1). This effect remained significant after full adjustment, and was accompanied by a significant interaction of CMV status by glycaemic status with EMRA levels $(p=0.031)$. In addition, these effects did not appear to be caused by the small group of diabetic individuals because identical results were found when diabetic participants were excluded from the analyses (data not shown). Analyses adjusted for age and sex showed that there were more EM T cells in hyperglycaemic individuals than in the normoglycaemic group, but this did not reach significance.

Increased $\mathrm{HbA}_{1 \mathrm{c}}$ is associated with increased $\mathrm{dCTL}$ numbers Table 3 shows the linear associations of metabolic factors with dCTL numbers. Overall, higher levels of continuous $\mathrm{HbA}_{1 \mathrm{c}}$ were associated with increased numbers of dCTLs (EM: $B=1.87, p<0.001$; EMRA: $B=2.05, p<0.01$ ) after adjustment for age, sex, marital status and SES (i.e. job status and manual occupation; Model 2). These associations remained significant after additional adjustment for lifestyle factors (Model 3; both $p<0.05$ ). The fasting glucose level was nonsignificantly associated with dCTL numbers in the overall sample (Table 3 ). When analyses were stratified by CMV status, the relationship between $\mathrm{HbA}_{1 \mathrm{c}}$ and increased dCTL numbers remained in $\mathrm{CMV}^{+}$, but not in $\mathrm{CMV}^{-}$individuals (both $p<0.01$ ).

$\mathrm{CMV}^{-}$and $\mathrm{HbA}_{1} \mathrm{c}^{- \text {-related associations between }}$ dyslipidaemia factors and dCTL numbers For all participants, linear regression showed that higher continuous levels of triacylglycerol were associated with increased numbers of

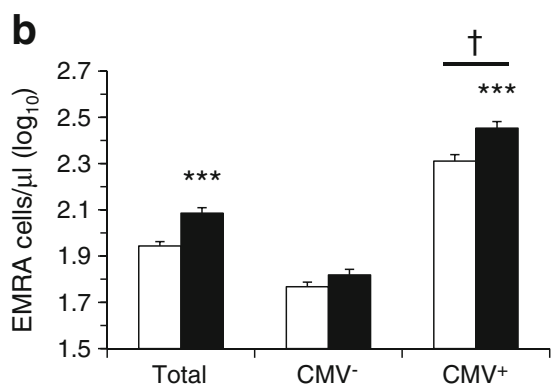

hyperglycaemic. $* p<0.05$ and $* * * p<0.001$ represent levels of significant difference from normoglycaemic. ${ }^{\dagger} p<0.001$ represents significant difference from $\mathrm{CMV}^{-}$

dCTLs after adjustment for confounders (Models 1-3; Table 3). Lower HDL-C levels and a higher LDL-C/HDL-C ratio were also significantly associated with increased $\mathrm{EM}$ and EMRA numbers after adjustment for sociodemographic factors (Model 2). Except for the LDL-C/HDL-C association with EM T cells, these relationships were attenuated by additional adjustment for lifestyle factors (Model 3). Total cholesterol and LDL-C levels were nonsignificantly associated with dCTL numbers in the full sample (Table 3).

When analyses were stratified by CMV status, the relationship between triacylglycerol and increased dCTL numbers was found only in $\mathrm{CMV}^{+}$individuals. Additionally, in the $\mathrm{CMV}^{+}$group, total cholesterol and the LDL-C/HDL-C ratio were positively associated with EM T cell numbers after adjustment for sociodemographic factors (Model 2; both $p<0.05$ ), although only total cholesterol remained significant after full adjustment (Model 3; Table 3).

To test the influence of glucose metabolism on the significant lipid profile associations in $\mathrm{CMV}^{+}$individuals (i.e. triacylglycerol and cholesterol), $\mathrm{HbA}_{1 \mathrm{c}}$ was additionally entered into each of these fully adjusted models. After the addition of $\mathrm{HbA}_{1 \mathrm{c}}$ to the model, the associations between triacylglycerol and $\mathrm{dCTL}$ were attenuated, while the relationship between cholesterol and EM T cells remained significant (data not shown). Among CMV individuals, no significant associations were observed between any metabolic factors and dCTL numbers (Table 3).

\section{Discussion}

A hallmark of an ageing immune system is the accumulation of differentiated $\mathrm{CD}^{+} \mathrm{T}$ cells, which is strongly enhanced in CMV-infected individuals. The present study demonstrated that impaired glycaemic control, as measured by $\mathrm{HbA}_{1 \mathrm{c}}$ levels, is associated with elevated numbers of dCTL in $\mathrm{CMV}^{+}$individuals, but not in $\mathrm{CMV}^{-}$individuals. This association was robust to adjustment for demographic, SES and lifestyle factors. Thus, this study is the first to provide evidence that glycaemic control may contribute to 
Table 3 Unstandardised coefficients from linear regressions of metabolic factors and EM and EMRA T cell numbers

\begin{tabular}{|c|c|c|c|c|c|c|}
\hline \multirow[t]{2}{*}{ Variables } & \multicolumn{3}{|c|}{$\mathrm{EM}\left(\mathrm{CD} 27^{-} \mathrm{CD}^{2} 4 \mathrm{RA}^{-}\right)$} & \multicolumn{3}{|c|}{ EMRA $\left(\mathrm{CD} 27^{-} \mathrm{CD}^{2} 5 \mathrm{RA}^{+}\right)$} \\
\hline & Model 1 & Model 2 & Model 3 & Model 1 & Model 2 & Model 3 \\
\hline \multicolumn{7}{|c|}{ All participants $(n=1,103)$} \\
\hline $\mathrm{HbA}_{1 \mathrm{c}}$ & $2.03(0.58)^{* * *}$ & $1.87(0.59)^{* * *}$ & $1.54(0.59)^{*}$ & $2.20(0.77)^{* *}$ & $2.05(0.77)^{* *}$ & $1.68(0.78)^{*}$ \\
\hline Fasting glucose & $2.15(2.06)$ & $2.31(2.06)$ & $3.23(2.07)$ & $-2.92(2.69)$ & $-2.74(2.70)$ & $-1.72(2.73)$ \\
\hline Cholesterol & $0.01(0.01)$ & $0.01(0.01)$ & $0.01(0.01)$ & $0.01(0.01)$ & $0.01(0.01)$ & $0.02(0.01)$ \\
\hline LDL-C & $0.01(0.01)$ & $0.01(0.01)$ & $0.01(0.01)$ & $0.01(0.01)$ & $0.01(0.01)$ & $0.01(0.01)$ \\
\hline HDL-C & $-0.13(0.05)^{* *}$ & $-0.13(0.05)^{* *}$ & $-0.06(0.05)$ & $-0.16(0.06)^{* *}$ & $-0.15(0.06)^{*}$ & $-0.08(0.06)$ \\
\hline LDL-C/HDL-C & $0.20(0.07)^{* *}$ & $0.20(0.07)^{* *}$ & $0.14(0.07)^{*}$ & $0.21(0.09)^{*}$ & $0.21(0.09)^{*}$ & $0.13(0.10)$ \\
\hline Triacylglycerol & $0.14(0.05)^{* *}$ & $0.13(0.05)^{* *}$ & $0.11(0.05)^{*}$ & $0.21(0.07)^{* *}$ & $0.20(0.07)^{* *}$ & $0.18(0.07)^{*}$ \\
\hline \multicolumn{7}{|c|}{ CMV negative $(n=703)$} \\
\hline $\mathrm{HbA}_{1 \mathrm{c}}$ & $0.97(0.65)$ & $0.86(0.65)$ & $0.46(0.66)$ & $0.87(0.23)$ & $0.94(0.82)$ & $0.48(0.84)$ \\
\hline Fasting glucose & $2.34(2.18)$ & $2.40(2.18)$ & $3.75(2.23)$ & $-1.3(2.77)$ & $-1.29(2.77)$ & $0.36(2.84)$ \\
\hline Cholesterol & $0.00(0.01)$ & $0.00(0.01)$ & $0.00(0.01)$ & $0.01(0.01)$ & $0.01(0.01)$ & $0.01(0.01)$ \\
\hline LDL-C & $0.00(0.01)$ & $0.01(0.01)$ & $0.00(0.01)$ & $0.01(0.01)$ & $0.01(0.01)$ & $0.01(0.01)$ \\
\hline HDL-C & $-0.09(0.05)$ & $-0.08(0.05)$ & $-0.03(0.05)$ & $-0.02(0.06)$ & $-0.03(0.06)$ & $0.02(0.07)$ \\
\hline LDL-C/HDL-C & $0.11(0.07)$ & $0.12(0.07)$ & $0.06(0.08)$ & $0.08(0.09)$ & $0.09(0.09)$ & $0.01(0.10)$ \\
\hline Triacylglycerol & $0.07(0.06)$ & $0.06(0.06)$ & $0.03(0.06)$ & $0.12(0.07)$ & $0.13(0.07)$ & $0.09(0.07)$ \\
\hline \multicolumn{7}{|l|}{ CMV positive $(n=400)$} \\
\hline $\mathrm{HbA}_{1 \mathrm{c}}$ & $3.11(1.03)^{* *}$ & $3.02(1.03)^{* *}$ & $2.75(1.03)^{* *}$ & $3.02(1.00)^{* *}$ & $2.92(1.01)^{* *}$ & $2.90(1.02)^{* *}$ \\
\hline Fasting glucose & $2.41(3.82)$ & $2.19(3.83)$ & $2.36(3.83)$ & $-3.86(3.72)$ & $-3.78(3.74)$ & $-3.40(3.77)$ \\
\hline Cholesterol & $0.04(0.02)^{*}$ & $0.04(0.02)^{*}$ & $0.04(0.02)^{*}$ & $0.03(0.02)$ & $0.03(0.02)$ & $0.03(0.02)$ \\
\hline LDL-C & $0.03(0.01)$ & $0.03(0.01)$ & $0.03(0.01)^{*}$ & $0.02(0.01)$ & $0.02(0.01)$ & $0.02(0.01)$ \\
\hline HDL-C & $-0.11(0.08)$ & $-0.10(0.08)$ & $-0.04(0.09)$ & $-0.11(0.08)$ & $-0.10(0.08)$ & $-0.06(0.09)$ \\
\hline LDL-C/HDL-C & $0.27(0.12)^{*}$ & $0.26(0.12)^{*}$ & $0.22(0.13)$ & $0.21(0.12)$ & $0.22(0.12)$ & $0.18(0.13)$ \\
\hline Triacylglycerol & $0.22(0.09) *$ & $0.22(0.09)^{*}$ & $0.20(0.10)^{*}$ & $0.23(0.09)^{*}$ & $0.22(0.09)^{*}$ & $0.21(0.10)^{*}$ \\
\hline
\end{tabular}

Data are unstandardised coefficients (B) (SEM)

Model 1: adjusted for age and sex

Model 2: model 1 with additional adjustment for marital status and SES (job status and manual occupation)

Model 3: model 2 further adjusted for smoking, alcohol, BMI and physical activity

${ }^{*} p<0.05, * * p<0.01$ and $* * * p=0.001$

immunosenescence by amplifying the effects of CMV on T cell differentiation. This mechanism may contribute to the impaired immunity seen in hyperglycaemia and diabetic patients, and may possibly be a pathway linking CMV to increased CVD risk.

$\mathrm{HbA}_{1 \mathrm{c}}$ may be positively associated with $\mathrm{dCTL}$ in $\mathrm{CMV}^{+}$ individuals due to a synergistic impact on both the extent of CMV replication and the $\mathrm{T}$ cell response to CMV activity. Elevated glucose levels may: (1) directly and indirectly increase the efficiency and frequency of CMV replication; and (2) enhance the T cell responses to CMV. First, CMV-induced upregulation of GLUT4 enhances glucose uptake [33]. This excess glucose influx is diverted towards the biosynthesis of fatty acids, which are used to directly increase viral production and enhance infectivity [34]. Indirectly, elevated glucose promotes the production of reactive oxygen species, which are known to stimulate the CMV promoter region and provide the first step that is necessary, but not sufficient, for the reactivation of CMV [35, 36]. Second, evidence from in vitro studies demonstrates that strong, repeated antigen receptor stimulation (e.g. by CMV) can lead to the upregulation of GLUT1 and to enhanced glucose uptake by $\mathrm{T}$ cells. Excess glucose uptake was shown to parallel increased T cell activation, proinflammatory cytokine production and an elevated threshold for cell death [21, 22]. Although not directly investigated in the present study, these represent biologically plausible mechanisms through which elevated glucose could augment dCTL numbers via CMV activity.

Attenuation of the relationship between hyperglycaemia and CMV infection by sociodemographic factors is in line with the finding of null associations after similar adjustments in other studies $[37,38]$, but is inconsistent with the somewhat divergent epidemiological data from Chen and colleagues in very old (85 years) adults [39]. The former finding suggests 
that unadjusted differences in glucose by CMV status are likely to be a consequence of common predisposing factors, rather than being causally linked. Indeed, both CMV infection and dysregulated glucose metabolism are more common in individuals with a variety of pre-existing health risk factors. For example, CMV has a non-random distribution in the population: infection is particularly prevalent among those who are typically older, current smokers or have low SES (job status, education) $[31,40]$. On the other hand, the robust linear relationships between $\mathrm{HbA}_{1 \mathrm{c}}$ and both EM and EMRA T cells found only in $\mathrm{CMV}^{+}$individuals suggest that glycaemic status may contribute to immune responses to CMV reactivation rather than to initial infection.

The current positive relationship between markers of dyslipidaemia and numbers of dCTLs in $\mathrm{CMV}^{+}$individuals appears to be partly a by-product of the intrinsic link between $\mathrm{HbA}_{1 \mathrm{c}}$ level and lipid metabolism found in both diabetic [23] and non-diabetic adults [41]. Evidence for this relationship is provided by the null associations between triacylglycerol and $\mathrm{dCTL}$ numbers in $\mathrm{CMV}^{+}$individuals after additional adjustment for $\mathrm{HbA}_{1 \mathrm{c}}$ level in the current study. In contrast, the cholesterol-EM association remained after additional adjustment for $\mathrm{HbA}_{1 \mathrm{c}}$ level, making a more direct effect of CMV more plausible. The factors underlying this association are not clear, but could reflect the manifestation of a host defence mechanism to limit CMV infectivity. At an early stage of CMV infection, cells increase the expression of CD91, which regulates lipid metabolism and decreases intracellular cholesterol [42]. As intracellular cholesterol is necessary for enhanced CMV virus production and effective entry into other cells $[43,44]$, a reduction in cellular cholesterol uptake by the host cell could explain the association between elevated cholesterol and EM accumulation seen here [42]. This does not, however, rule out the possibility of CMV-induced alterations in systemic lipid and glucose levels.

The current finding that $\mathrm{CMV}^{+}$individuals with higher levels of $\mathrm{HbA}_{1 \mathrm{c}}$ and CVD risk factors (cholesterol and triacylglycerol) had elevated dCTLs provides further evidence for a common impact of both CMV [30, 45, 46] and dysregulated glucose metabolism [47] on cardiac health. Consistent with this notion, studies have reported associations among circulating dCTL, CMV-specific T cell responses and increased CVD risk factors, such as heart valve calcification, carotid artery thickness and increased BP [48-50]. Taken together, these findings support the hypothesis proposed by Simanek and colleagues [32] that CMV infection and inflammation partially impact mortality risk via their combined contribution to other CVD risk factors, and further suggest dysregulated glucose metabolism and increased dCTLs as additional mechanisms.

There are a number of limitations with the current study that should be acknowledged. First, the low number of diabetic participants precluded their inclusion as a separate group; comparisons were instead performed between normoglycaemic and a merged hyperglycaemic group. Information about whether these participants had type 1 or type 2 diabetes was also unavailable; however, in the general population, type 1 diabetes represents only $5-10 \%$ of the diabetes cases [27]. Likewise, clinically relevant markers of immunosenescence (e.g. short telomere length) are observed even at the early stages of glucose dysregulation [4, 20], and the associations found in hyperglycaemic individuals were not altered by removal of the diabetic group from the analyses. Second, there was no direct measure of subsequent CMV reactivation. Nevertheless, the selective EMRA T cell accumulation is almost exclusively associated with CMV infection, and there is a strong empirical basis to suggest that these associations reflect CMV activity [2, 6, 9]. However, future studies should include more direct measures of CMV reactivation (e.g. quantitative CMV-specific antibody levels) for comparison. Finally, because of the cross-sectional nature of the study, we are unable to discern cause-effect relationships. Given the complex interplay between components of the immune system, metabolic factors, CMV and ageing, as well as the contribution of potential intermediaries such as oxidative stress and inflammation to these processes, bidirectional or cyclical relationships between these factors cannot be ruled out.

In conclusion, we observed associations between measures of glucose metabolism (i.e. $\mathrm{HbA}_{1 \mathrm{c}}$ level and diabetic status), dyslipidaemia (total cholesterol and triacylglycerol levels) and $\mathrm{dCTL}$ subsets in $\mathrm{CMV}^{+}$individuals. These associations with $\mathrm{HbA}_{1 \mathrm{c}}$ level withstood adjustment for demographic, SES and lifestyle factors known to impact both CMV infection and glucose metabolism, thus demonstrating a robust association. Overall, it appears that these metabolic factors act reciprocally with CMV to amplify the accumulation of EM and EMRA $\mathrm{CD}^{+} \mathrm{T}$ cells, and represent potentially biologically relevant pathways underlying the CMV-induced acceleration of immunosenescence. These data also highlight CMV and dCTL accumulation as a potentially overlooked mechanism underlying the associations of hyperglycaemia and diabetes with impaired immunity. These links to immunosenescence are particularly relevant in the context of increased incidence of type 2 diabetes and an associated defective viral response in an ageing population.

Acknowledgements Personnel from Health Vision Ltd. (Berlingen, Switzerland) performed the company health check used in the current study. The authors are indebted to I. Gergei (Ruprecht Karls University Heidelberg, Mannheim, Germany), T. Rausch (Mannheim Institute for Public Health, Heidelberg University, Germany) and L. Rotar (Health Vision, Berlingen, Switzerland) for their indispensable assistance in immunological data collection.

Funding This work was funded by a Marie Curie Initial Training Network award (ITN-2009-238665 NINA).

Duality of interest JEF was the major shareholder of Health Vision Ltd., which organised the data collection in 2011, until 31 December 2012. Since 1 January 2013, he has served as a scientific consultant to 
Health Vision Ltd. The remaining authors declare that there is no duality of interest associated with this manuscript.

Contribution statement All of the authors helped with the conception and design of the study, as well as the analysis and/or interpretation of the data. All authors assisted in drafting the article and/or revising it critically. JLR contributed to data acquisition. All authors approved the final version of the manuscript for publication. JAB is the guarantor of this work.

Open Access This article is distributed under the terms of the Creative Commons Attribution 4.0 International License (http:// creativecommons.org/licenses/by/4.0/), which permits unrestricted use, distribution, and reproduction in any medium, provided you give appropriate credit to the original author(s) and the source, provide a link to the Creative Commons license, and indicate if changes were made.

\section{References}

1. Larbi A, Franceschi C, Mazzatti D, Solana R, Wikby A, Pawelec G (2008) Aging of the immune system as a prognostic factor for human longevity. Physiology 23:64-74

2. Turner JE, Campbell JP, Edwards KM et al (2014) Rudimentary signs of immunosenescence in Cytomegalovirus-seropositive healthy young adults. Age 36:287-297

3. Franceschi $\mathrm{C}$, Bonafè $\mathrm{M}$, Valensin $\mathrm{S}$ et al (2000) Inflamm-aging. An evolutionary perspective on immunosenescence. Ann N Y Acad Sci 908:244-254

4. Salpea KD, Humphries SE (2010) Telomere length in atherosclerosis and diabetes. Atherosclerosis 209:35-38

5. Sansoni P, Vescovini R, Fagnoni F et al (2008) The immune system in extreme longevity. Exp Gerontol 43:61-65

6. Chidrawar S, Khan N, Wei W et al (2009) Cytomegalovirusseropositivity has a profound influence on the magnitude of major lymphoid subsets within healthy individuals. Clin Exp Immunol 155:423-432

7. Dowd JB, Bosch JA, Steptoe A et al (2013) Cytomegalovirus is associated with reduced telomerase activity in the Whitehall II cohort. Exp Gerontol 48:385-390

8. van de Berg PJ, Griffiths SJ, Yong SL et al (2010) Cytomegalovirus infection reduces telomere length of the circulating $\mathrm{T}$ cell pool. $\mathrm{J}$ Immunol 184:3417-3423

9. van de Berg PJ, van Stijn A, Ten Berge IJ, van Lier RA (2008) A fingerprint left by cytomegalovirus infection in the human $\mathrm{T}$ cell compartment. J Clin Virol 41:213-217

10. Kuijpers TW, Vossen MT, Gent MR et al (2003) Frequencies of circulating cytolytic, CD45RA+CD27-, CD8+ T lymphocytes depend on infection with CMV. J Immunol 170:4342-4348

11. Messaoudi I, Lemaoult J, Guevara-Patino JA, Metzner BM, Nikolich-Zugich J (2004) Age-related CD8 T cell clonal expansions constrict CD8 T cell repertoire and have the potential to impair immune defense. J Exp Med 200:1347-1358

12. Macaulay R, Akbar AN, Henson SM (2013) The role of the T cell in age-related inflammation. Age 35:563-572

13. Akbar AN, Henson SM (2011) Are senescence and exhaustion intertwined or unrelated processes that compromise immunity? Nat Rev Immunol 11:289-295

14. Almanzar G, Schwaiger S, Jenewein B et al (2005) Long-term cytomegalovirus infection leads to significant changes in the composition of the CD8+ T-cell repertoire, which may be the basis for an imbalance in the cytokine production profile in elderly persons. $\mathrm{J}$ Virol 79:3675-3683
15. Allard R, Leclerc P, Tremblay C, Tannenbaum TN (2010) Diabetes and the severity of pandemic influenza A (H1N1) infection. Diabetes Care 33:1491-1493

16. Joshi N, Caputo GM, Weitekamp MR, Karchmer AW (1999) Infections in patients with diabetes mellitus. N Engl J Med 341: 1906-1912

17. Shah BR, Hux JE (2003) Quantifying the risk of infectious diseases for people with diabetes. Diabetes Care 26:510-513

18. Egawa Y, Ohfuji S, Fukushima W et al (2014) Immunogenicity of influenza $\mathrm{A}(\mathrm{H} 1 \mathrm{~N} 1)$ pdm09 vaccine in patients with diabetes mellitus: with special reference to age, body mass index, and HbA1c. Hum Vaccin Immunother 10:1187-1194

19. Esposito K, Nappo F, Marfella R et al (2002) Inflammatory cytokine concentrations are acutely increased by hyperglycemia in humans: role of oxidative stress. Circulation 106:2067-2072

20. Adaikalakoteswari A, Balasubramanyam M, Ravikumar R, Deepa R, Mohan V (2007) Association of telomere shortening with impaired glucose tolerance and diabetic macroangiopathy. Atherosclerosis 195:83-89

21. Maciver NJ, Jacobs SR, Wieman HL, Wofford JA, Coloff JL, Rathmell JC (2008) Glucose metabolism in lymphocytes is a regulated process with significant effects on immune cell function and survival. J Leukoc Biol 84:949-957

22. Zhao Y, Altman BJ, Coloff JL et al (2007) Glycogen synthase kinase 3alpha and 3beta mediate a glucose-sensitive antiapoptotic signaling pathway to stabilize Mcl-1. Mol Cell Biol 27:4328-4339

23. Khan HA, Sobki SH, Khan SA (2007) Association between glycaemic control and serum lipids profile in type 2 diabetic patients: HbA1c predicts dyslipidaemia. Clin Exp Med 7:24-29

24. Jönsson D, Rosengren A, Dotevall A, Lappas G, Wilhelmsen L (1999) Job control, job demands and social support at work in relation to cardiovascular risk factors in MONICA 1995, Göteborg. J Cardiovasc Risk 6:379-385

25. Sacks DB, Bruns DE, Goldstein DE, Maclaren NK, McDonald JM, Parrott M (2002) Guidelines and recommendations for laboratory analysis in the diagnosis and management of diabetes mellitus. Clin Chem 48:436-472

26. Friedewald WT, Levy RI, Fredrickson DS (1972) Estimation of the concentration of low-density lipoprotein cholesterol in plasma, without use of the preparative ultracentrifuge. Clin Chem 18:499502

27. American Diabetes A (2013) Diagnosis and classification of diabetes mellitus. Diabetes Care 36(Suppl 1):S67-S74

28. Grundy SM, Cleeman JI, Daniels SR et al (2005) Diagnosis and management of the metabolic syndrome: an American Heart Association/National Heart, Lung, and Blood Institute Scientific Statement. Circulation 112:2735-2752

29. Gould W, Hilbe J (1991) sed2. Ladder-of-Powers variable transformation. Stata Technical Bulletin July:14-15

30. Savva GM, Pachnio A, Kaul B et al (2013) Cytomegalovirus infection is associated with increased mortality in the older population. Aging Cell 12:381-387

31. Rector JL, Dowd JB, Loerbroks A et al (2014) Consistent associations between measures of psychological stress and CMV antibody levels in a large occupational sample. Brain Behav Immun 38:133141

32. Simanek AM, Dowd JB, Pawelec G, Melzer D, Dutta A, Aiello AE (2011) Seropositivity to cytomegalovirus, inflammation, all-cause and cardiovascular disease-related mortality in the United States. PLoS One 6:e16103

33. Yu Y, Maguire TG, Alwine JC (2011) Human cytomegalovirus activates glucose transporter 4 expression to increase glucose uptake during infection. J Virol 85:1573-1580

34. Spencer CM, Schafer XL, Moorman NJ, Munger J (2011) Human cytomegalovirus induces the activity and expression of acetyl- 
coenzyme a carboxylase, a fatty acid biosynthetic enzyme whose inhibition attenuates viral replication. J Virol 85:5814-5824

35. Cohen G, Riahi Y, Shamni O et al (2011) Role of lipid peroxidation and PPAR- in amplifying glucose-stimulated insulin secretion. Diabetes 60:2830-2842

36. Jaganjac M, Matijevic T, Cindric M et al (2010) Induction of CMV1 promoter by 4-hydroxy-2-nonenal in human embryonic kidney cells. Acta Biochim Pol 57:179-183

37. Haeseker MB, Pijpers E, Dukers-Muijrers NH et al (2013) Association of cytomegalovirus and other pathogens with frailty and diabetes mellitus, but not with cardiovascular disease and mortality in psycho-geriatric patients; a prospective cohort study. Immun Ageing 10:30

38. Lutsey PL, Pankow JS, Bertoni AG, Szklo M, Folsom AR (2009) Serological evidence of infections and type 2 diabetes: the MultiEthnic Study of Atherosclerosis. Diabet Med 26:149-152

39. Chen S, de Craen AJ, Raz Y et al (2012) Cytomegalovirus seropositivity is associated with glucose regulation in the oldest old. Results from the Leiden 85-plus Study. Immun Ageing 9:18

40. Dowd JB, Aiello AE, Alley DE (2009) Socioeconomic disparities in the seroprevalence of cytomegalovirus infection in the US population: NHANES III. Epidemiol Infect 137:58-65

41. Selvin E, Steffes MW, Zhu H et al (2010) Glycated hemoglobin, diabetes, and cardiovascular risk in nondiabetic adults. N Engl J Med 362:800-811

42. Gudleski-O'Regan N, Greco TM, Cristea IM, Shenk T (2012) Increased expression of LDL receptor-related protein 1 during human cytomegalovirus infection reduces virion cholesterol and infectivity. Cell Host Microbe 12:86-96
43. Chukkapalli V, Heaton NS, Randall G (2012) Lipids at the interface of virus-host interactions. Curr Opin Microbiol 15:512-518

44. Williamson CD, Zhang A, Colberg-Poley AM (2011) The human cytomegalovirus protein UL37 exon 1 associates with internal lipid rafts. J Virol 85:2100-2111

45. Gkrania-Klotsas E, Langenberg C, Sharp S, Luben R, Khaw K-T, Wareham N (2012) Higher immunoglobulin G antibody levels against cytomegalovirus are associated with incident ischemic heart disease in the population-based EPIC-Norfolk cohort. J Infect Dis 206:1897-1903

46. van de Berg PJ, Yong S-L, Remmerswaal EB, van Lier RA, ten Berge IJ (2012) Cytomegalovirus-induced effector T cells cause endothelial cell damage. Clin Vaccine Immunol 19:772-779

47. Barr EL, Boyko EJ, Zimmet PZ, Wolfe R, Tonkin AM, Shaw JE (2009) Continuous relationships between non-diabetic hyperglycaemia and both cardiovascular disease and all-cause mortality: the Australian Diabetes, Obesity, and Lifestyle (AusDiab) study. Diabetologia 52:415-424

48. Hsue PY, Hunt PW, Sinclair E et al (2006) Increased carotid intimamedia thickness in HIV patients is associated with increased cytomegalovirus-specific T-cell responses. Aids 20:2275-2283

49. Terrazzini N, Bajwa M, Vita S et al (2014) A novel cytomegalovirus-induced regulatory-type T-cell subset increases in size during older life and links virus-specific immunity to vascular pathology. J Infect Dis 209:1382-1392

50. Winchester R, Wiesendanger M, O'Brien W et al (2011) Circulating activated and effector memory $\mathrm{T}$ cells are associated with calcification and clonal expansions in bicuspid and tricuspid valves of calcific aortic stenosis. J Immunol 187:1006-1014 